\title{
Study on the Effect of Plasma Treatment on Flat-band-voltage and Equivalent Oxide Thickness using Metal-organic Chemical Vapor Deposition TiN Film as p-MOSFETS Metal Gate Electrode
}

Jianfeng Gao*, Hong Yang, Guobin Bai, Junfeng Li and Chao Zhao

Institute of Microelectronics Chinese Academy of Sciences, No. 3, Bei-Tu-Cheng West Road, Beijing, 100029, China

\begin{abstract}
This work aimed to study on the effects of plasma treatment on flat band voltage $(\mathrm{Vfb})$ and equivalent oxide thickness (EOT) using Metal-organic Chemical Vapor Deposition (MOCVD) TiN film as p-MOSFETs metal gate electrode. The plasma treatment conditions effect on the resistance and composition properties of MOCVD TiN, consequently, they can modulate work function and control threshold voltage. The effects of "plasma treatment" were imposed to favor the formation of Ti-N bonds, thus decreasing the rate Ti-C bond and favoring a crystallized stoichiometric TiN phase to increase PMOS Vfb shift. Meanwhile, plasma treatments accelerate interfacial oxide formation and increase EOT. On the other hand, EOT increases with the increase of plasma treatment time and power in this work, but Vfb does not always increase and reach a max value.
\end{abstract}

Keywords: MOCVD TiN; EOT; Vfb; Plasma treatment; pMOSFETs

Abbreviations: Vfb: Flat-Band-Voltage; EOT: Equivalent Oxide Thickness; MOCVD: Metal-Organic Chemical Vapor Deposition; MOS: Metal-Oxide-Semiconductor; TDMAT: Tetra (Dimethylamino) Titanium; XPS: X-Ray Photoelectron Spectrometer; AFM: Atomic Force Microscope; XRD: X-Ray Diffraction; TEMAH: Tetrakis (Ethylmethylamido) Hafnium; IL: Interfacial Layer; ALD: Atom Layer Deposition; HRTEM: High Resolution Transmission Electron Microscopy; FWHM: The Full Width Half Maximum (FWHM); RMS: Root Mean Square

\section{Introduction}

Scaling of sub- $45 \mathrm{~nm}$ complementary metal-oxide semiconductor CMOS devices requires metal gate electrodes to address restricting factors of usual poly silicon gates, such as poly depletion, doping species penetration, and Fermi level pinning [1,2]. However, integration of the high $\mathrm{K}$ dielectric/metal gate stack into a metal-oxide-semiconductor (MOS) transistor provides some new challenges, such as Vfb and EOT control $[3,4]$. The requirements for the metal gates are as follows: favorable work functions, low sheet resistance, thermal stability, and compatibility with high-k dielectric Among all the potential candidates for metal gate electrodes, TiN gates showed promising results such as thermal stability with the high-k gate dielectrics and process compatibility $[5,6]$. Currently, Titanium nitride (TiN) films deposited with atom layer deposition (ALD) and reactive sputter is widely studied as metal gate in semi-conductor technology [5-9]. In this work, TiN films were deposited by using a MOCVD technique. The Tetra (dimethylamino) titanium (TDMAT) precursor has been used to obtain TiN films. $\mathrm{N}_{2}$ and $\mathrm{H}_{2}$ plasma treatment after TiN thin film growth modifies film composition and physical properties. Capacitors with $\mathrm{W} / \mathrm{TiN}$ gate stacks are fabricated for Vfb and EOT extraction, effects of TiN growth time, plasma treatment time and power on the work function and EOT are systematically investigated.

\section{Experimental}

In this work, the depositions of MOCVD-TiN films were performed in an Endura ${ }^{\mathrm{am}} 5500$ tool produced by Applied Materials, Inc. During the MOCVD TiN deposition, the temperature was controlled at $400 \pm 2^{\circ} \mathrm{C}$. A blanket MOCVD TiN film was deposited with different conditions including growth time, plasma treatment time and power. In the $\mathrm{H}_{2} / \mathrm{N}_{2}$ plasma treatment, the flow rates of $\mathrm{H}_{2}$ and $\mathrm{N}_{2}$ gases were $450 \mathrm{sccm}$ and $300 \mathrm{sccm}$, respectively. The process pressures of the TiN deposition and the plasma treatment were at 5 torr and 1.3 torr, respectively. The sheet resistance of blanket TiN films was analyzed by a four-point probe. The film properties of MOCVD TiN films were examined by X-Ray Photoelectron Spectrometer (XPS), Atomic Force Microscope (AFM) and X-Ray Diffraction (XRD).

A MOS capacitor structure of metal $\mathrm{SiO}_{2} / \mathrm{p}$-type $\mathrm{Si}$ substrate was fabricated as shown in Figure 1a. Interfacial layer (IL) was grown to $\sim 0.8 \mathrm{~nm}$ with ozone chemical. $2 \mathrm{~nm} \mathrm{HfO}$ film was deposited on gate oxide using Tetrakis (ethylmethylamido) hafnium (TEMAH) as the precursor and $\mathrm{H}_{2} \mathrm{O}$ as the oxidant at $300^{\circ} \mathrm{C}$ in TFS200, then, these sample underwent post dielectric annealing under $450^{\circ} \mathrm{C}, 15 \mathrm{~s}$ at $\mathrm{N}_{2}$ ambience with a trace of $\mathrm{O}_{2}$ at atmospheric pressure. TiN with $2 \mathrm{~nm}$ as
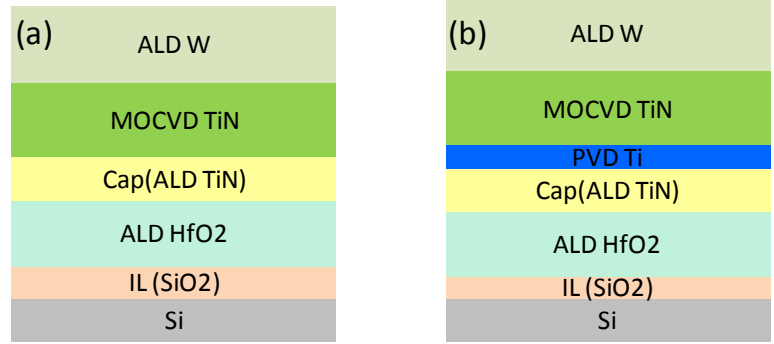

Figure 1: Schematic of the MOS capacitor structure.

*Corresponding author: Jianfeng Gao, Institute of Microelectronics Chinese Academy of Sciences, No. 3, Bei-Tu-Cheng West Road, Beijing, 100029, China, Tel: +86-10-8299578; E-mail: gaojianfeng@ime.ac.cn

Received November 25, 2015; Accepted December 16, 2015; Published December 26, 2015

Citation: Gao J, Yang H, Bai G, Li J, Zhao C (2015) Study on the Effect of Plasma Treatment on Flat-band-voltage and Equivalent Oxide Thickness using Metalorganic Chemical Vapor Deposition TiN Film as p-MOSFETS Metal Gate Electrode. J Nanomedic Nanotechnol S7:005. doi:10.4172/2157-7439.S7-005

Copyright: (c) $2015 \mathrm{Gao} \mathrm{J}$, et al. This is an open-access article distributed under the terms of the Creative Commons Attribution License, which permits unrestricted use, distribution, and reproduction in any medium, provided the original author and source are credited. 
Citation: Gao J, Yang H, Bai G, Li J, Zhao C (2015) Study on the Effect of Plasma Treatment on Flat-band-voltage and Equivalent Oxide Thickness using Metal-organic Chemical Vapor Deposition TiN Film as p-MOSFETS Metal Gate Electrode. J Nanomedic Nanotechnol S7:005. doi:10.4172/2157-7439.S7-005

capping layer was deposited on high-k layer. Next MOCVD TiN metal gates with different process conditions, which will be detailed in the following section, were deposited with Endura 5500 as work function layers. $750 \AA$ ALD W cap layers were then deposited for all the samples. Finally, after back oxide removal, $\mathrm{Al}$ metallization and the formation of gas annealing are performed. Moreover, another MOS capacitor is fabricated as shown in Figure 1b, Ti O-scavenging layer was deposited between ALD TiN cap layer and MOCVD TiN film. Vfb and EOT for the capacitors were extracted from high frequency capacitance-voltage (C-V) measurements with a Keithley 4200-SCS analyzer at $1 \mathrm{MHz}$. High Resolution Transmission Electron Microscopy (HRTEM) was carried out to characterize the physical properties.

\section{Results and Discussion}

Figure 2 shows the impacts of plasma treatment on MOCVD TiN film resistance. As shown in Figure 2, there is very obvious impact on film resistance with plasma treatment, film resistance will obvious decrease with increasing plasma treatment time and power. At the same condition of plasma treatment time, the resistance of higher plasma power treatment is lower than the lower condition; when plasma treatment time reaches or exceeds $35 \mathrm{~s}$, there is very little difference among $250 \mathrm{~W}, 400 \mathrm{~W}$ and $750 \mathrm{~W}$ plasma treatment conditions. XRD analysis results are shown in Figure 3 with different plasma treatment time at plasma treatment power of $400 \mathrm{~W}$ condition. It can be seen

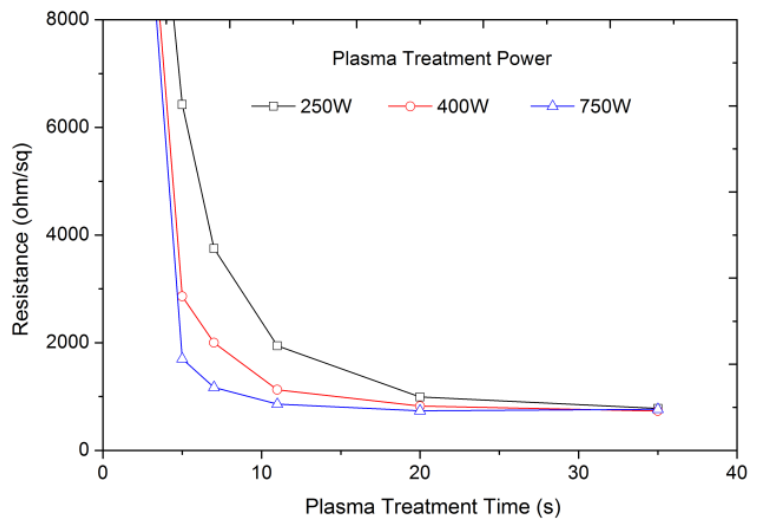

Figure 2: Impacts of plasma treatment time on MOCVD TiN resistance.

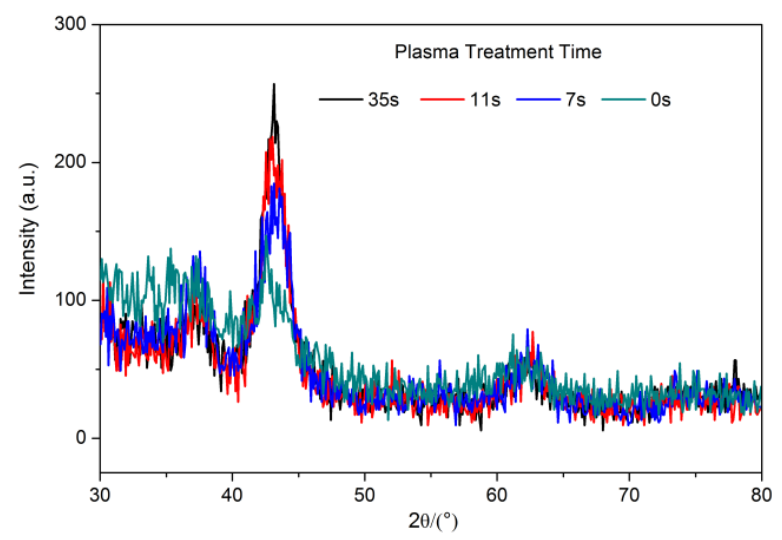

Figure 3: XRD analysis on MOCVD TiN film at various plasma treatment times.

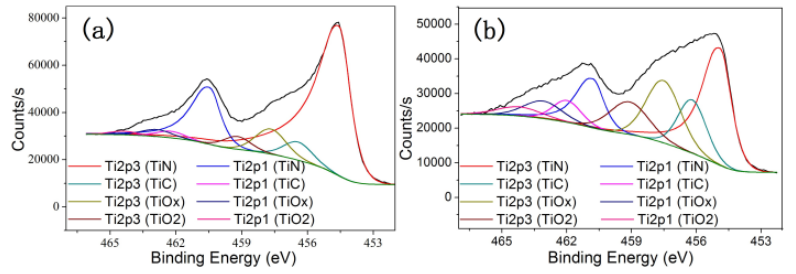

Figure 4: XPS analysis on MOCVD TiN film with (a) and without (b) plasma treatment.
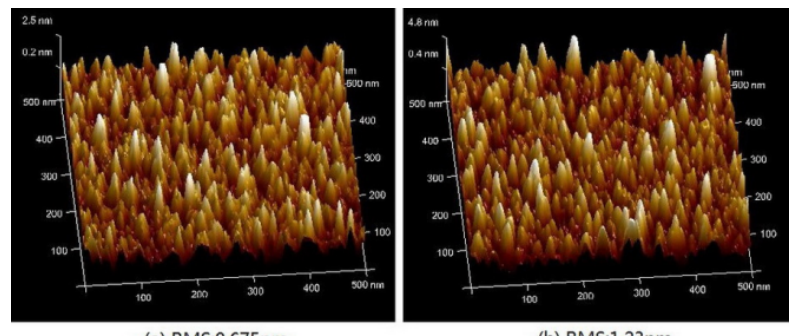

(a) RMS:0.675nm

(b) RMS:1.23nm

Figure 5: AFM analysis on MOCVD TiN film with (a) and without (b) plasma treatment.

that the diffraction intensity is very weak without plasma treatment condition, and extending plasma treatment time makes MOCVD TiN film (200) preferential orientation more remarkable and the full width half maximum (FWHM) of (002) diffraction more narrow, it is known that the FWHM obtained from the result of XRD relates to finite crystallite sizes, stacking faults, micro twins, stresses, dislocations, concentration variations, which parameters have a strong dependence on the crystal quality of films [10]. This suggests that plasma treatment make atmosphere TiN film including high $\mathrm{C}$ and $\mathrm{O}$ concentration crystallize and form strong Ti-N bonds. The chemical composition of MOCVD TiN film was analyzed by X-Ray Photoelectron Spectrometer (XPS), the Ti $i_{2}$ p peak can be fitted by eight Gaussian functions as Figure 4 shown, the XPS spectrum of $\mathrm{Ti}_{2} \mathrm{p}$ shows obviously different with and without plasma treatment. Compared with untreated MOCVD TiN films, $\mathrm{Ti}_{2} \mathrm{p}(\mathrm{TiN})$ ratio obviously increases and $\mathrm{Ti}_{2} \mathrm{p}(\mathrm{TiC}, \mathrm{TiOx}$ and $\mathrm{TiO}_{2}$ )ratio decrease with plasma treatment, it is helpful to form Ti-N bonds using plasma treatment, plasma treatment decreases the Ti-C and $\mathrm{Ti}-\mathrm{O}$ bond and favors a crystallized stoechiometric TiN phase. This result is matched with XRD analysis result. Figure 5 shows RMS roughness of MOCVD TiN with and without plasma treatment. It can be seen that plasma treatment can improve obviously MOCVD TiN film roughness which can be related with crystallization of TiN film due to decrease of $\mathrm{C}$ and O level observed on Figure 4a XPS result.

In order to investigate the effect of MOCVD TiN thickness on its work function, MOCVD TiN films with four different growth time (0 s, $4.1 \mathrm{~s}, 7 \mathrm{~s}, 15 \mathrm{~s}$ ) were deposited, growth time $0 \mathrm{~s}$ condition do not treat with plasma, other three conditions treated with plasma(power $750 \mathrm{~W}$ and time $35 \mathrm{~s}$ ). MOS capacitors are fabricated, and the $\mathrm{C}-\mathrm{V}$ characteristics were measured for $\mathrm{Vfb}$ and EOT extraction. Figure 6 shows the impacts of MOCVD TiN film growth time on Vfb and EOT. $\mathrm{Vfb}$ shifts positively with the increase of MOCVD TiN growth time, the Vfb of growth $7 \mathrm{~s}$ MOCVD TiN film is closely with growth $15 \mathrm{~s}$ MOCVD TiN film. This means that there is a critical thickness for the work function of MOCVD TiN film, it will stabilize over this critical 
Citation: Gao J, Yang H, Bai G, Li J, Zhao C (2015) Study on the Effect of Plasma Treatment on Flat-band-voltage and Equivalent Oxide Thickness using Metal-organic Chemical Vapor Deposition TiN Film as p-MOSFETS Metal Gate Electrode. J Nanomedic Nanotechnol S7:005. doi:10.4172/2157-7439.S7-005

Page 3 of 4

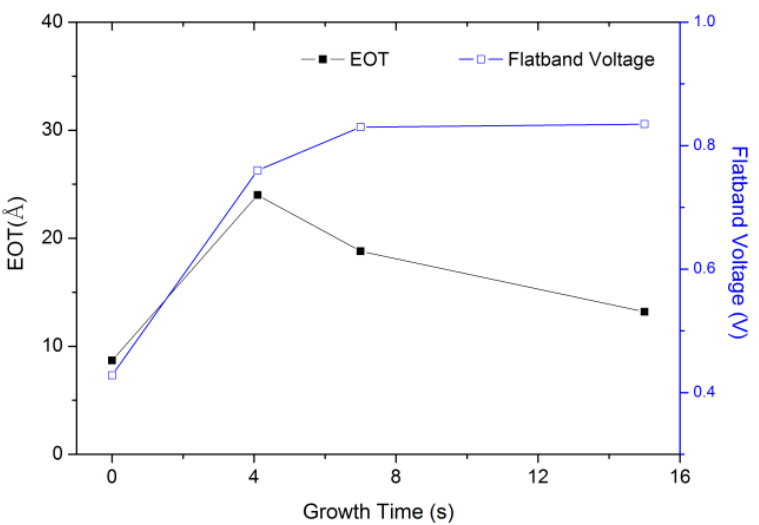

Figure 6: Impacts of MOCVD TiN growth time on EOT and Vfb.

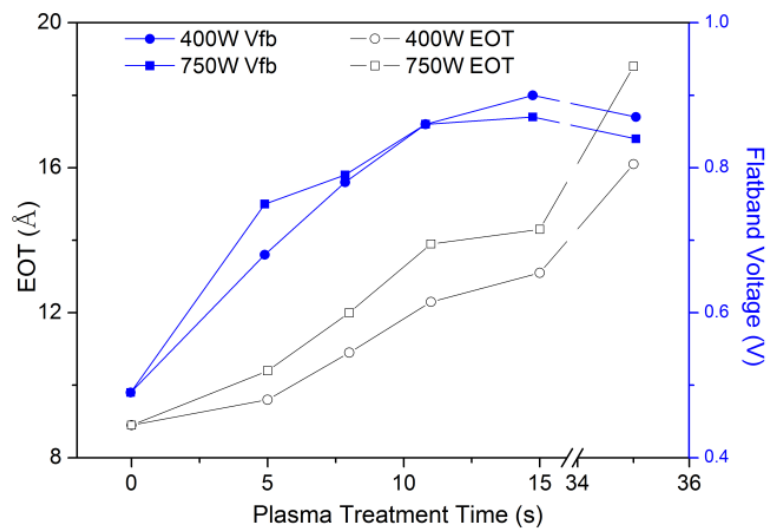

Figure 7: Impacts of MOCVD TiN plasma treatment time on EOT and Vfb.

value. It is reported that amorphous and laminated film has a lower work function than its crystalline and bulk counterpart due to densely packed crystallographic surfaces and therefore fewer broken surface bonds [11-14]. As the thickness of TiN increase, the crystallinity increases until it reaches a critical thickness, then the work function of TiN will be constant due to stabilizing crystallinity [15]. It can also be seen from Figure 6 that the EOT decreases obviously with increasing growth time of MOCVD TiN film, which may be related with oxygen diffusion ability through $\mathrm{HfO}_{2} / \mathrm{TiN}$ film into interface layer, it will be detailed in the following section.

MOCVD TiN films were deposited with different plasma treatment times $(0 \mathrm{~s}, 5 \mathrm{~s}, 8 \mathrm{~s}, 11 \mathrm{~s}, 15 \mathrm{~s}, 35 \mathrm{~s})$ and power $(400 \mathrm{~W}$ and $750 \mathrm{~W})$ so as to investigate the effect of plasma treatment on MOCVD TiN film work function. MOS capacitors are fabricated, and the $\mathrm{C}-\mathrm{V}$ characteristics were measured for $\mathrm{Vfb}$ and EOT extraction. As shown in Figure 7, there is max value of the work function of MOCVD TiN film with increasing plasma treatment time. This is because the work function of MOCVD TiN is not only related with crystallinity, but also related with grain size. Materials with smaller grain sizes have more grain boundaries, free electrons could be trapped in the grain boundaries and result in higher work function [13]. So work function of MOCVD TiN films will reach max value when films are fully crystallized, then maybe slightly decrease as film grains grow with further increasing plasma treatment time. It can also be seen from Figure 7 that EOT increases obviously with increasing plasma treatment time, and the impact of high power on EOT is more obvious than low power. Next, the impacts of plasma treatment on EOT will be discussed in detail.

Figure 8 shows the HRTEM images of samples as listed in Table 1. It can be seen that the $\mathrm{SiO}_{2}$-like interfacial layer (IL) of sample (a) is the thickest in all samples, the IL thickness of samples (c and d) is closely with initial $0.8 \mathrm{~nm}$ thickness, and the IL thickness of sample (b) is thicker than sample ( $c$ and d), but thinner than sample (a). The model of the effect of plasma treatment on EOT is schematically illustrated in Figure 9. It is well known that elements diffusion ability is related with diffusion coefficient D and concentration gradient according to Fick's first law of diffusion, and $\mathrm{D}$ is related with temperature. There is high concentration oxygen atom in plasma untreated MOCVD TiN film as shown in Figure 4b, the oxygen atoms of the film surface that gain energy from plasma have strong diffusion ability, and they are easily diffuse to $\mathrm{HfO}_{2}$ interface cross thin ALD TiN cap layer. The oxygen vacancies in the $\mathrm{HfO}_{2}$ layer act as mediators for oxygen atoms diffuse across the $\mathrm{HfO}_{2}$ into IL layer. These oxygen atoms react with substrate $\mathrm{Si}$ atom and form $\mathrm{SiO}_{2}$ that increase EOT of MOS capacitors (Figure 9a). It is reported that oxygen diffusion through amorphous $\mathrm{HfO}_{2}$ is quite rapid and this is not a rate-limiting step for this process [14]. Increasing MOCVD TiN thickness will increase oxygen atoms diffusion length and decrease concentration gradient of high energy oxygen atom; this will decrease oxygen atoms diffusion flux and decreases EOT of MOS capacitors (Figure 9b). This is consistent with our results in Figure 6, which shows the EOT decreases obviously with increasing growth time of MOCVD TiN film. In addition, increasing MOCVD TiN plasma treatment time will increase oxygen atom diffusion time, which makes more oxygen atoms diffuse into IL, this will increase EOT of MOS capacitors (Figure 7). Because oxygen atom cannot gain energy without plasma treatment and their diffusion will be suppressed (Figure 9c), it is very easy to explain why EOT of plasma untreated sample (c) is almost equal with initial IL thickness. Compared to the sample (a), there is thinner IL layer for sample (d) with Ti layer deposited between ALD TiN cap layer and MOCVD TiN film (Figure 8d), it was attributed to thin Ti metal film used as oxygen scavenging layer. In other words, Ti metal film acts as oxygen diffusion barrier and suppresses oxygen diffusion into IL layer (Figure 9d). From another point of view, it is proved that the increase of EOT with the increase of plasma treatment time and power is due to oxygen diffusion of MOCVD TiN film into IL layer.

\section{Summary and Conclusions}

Titanium nitride (TiN) films were prepared by using a metalorganic chemical vapor deposition (MOCVD) technique with varying growth and plasma treatment conditions. The plasma treatment conditions effect on the resistance and composition properties of MOCVD TiN film. The effects of plasma treatment were imposed to favor the formation of Ti-N bonds, thus decreasing the rate Ti-C bond and favoring a crystallized stoichiometric TiN phase to increase $\mathrm{Vfb}$ value as PMOS metal gate electrode. Meanwhile, plasma treatments
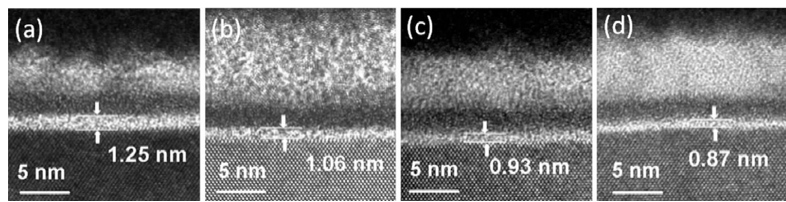

Figure 8: HRTEM images of samples as listed in table. 
Citation: Gao J, Yang H, Bai G, Li J, Zhao C (2015) Study on the Effect of Plasma Treatment on Flat-band-voltage and Equivalent Oxide Thickness using Metal-organic Chemical Vapor Deposition TiN Film as p-MOSFETS Metal Gate Electrode. J Nanomedic Nanotechnol S7:005. doi:10.4172/2157-7439.S7-005

Page 4 of 4

\begin{tabular}{|c|l|c|c|}
\hline Sample & Film stack (Top-down) & \multicolumn{2}{|c|}{ MOCVD TiN condition } \\
\cline { 3 - 4 } & & Growth time & Plasma \\
\hline a & ALD W/MOCVD TiN/ ALD TiN/ HfO2/IL/Si sub. & $7 s$ & Treatment power \\
\hline b & ALD W/MOCVD TiN/ ALD TiN/ HfO2/IL/Si sub. & 15s & $400 \mathrm{~W}$ \\
\hline c & ALD W/MOCVD TiN/ ALD TiN/ HfO2/IL/Si sub. & $7 \mathrm{~s}$ & $400 \mathrm{~W}$ \\
\hline d & ALD W/MOCVD TiN/PVD Ti/ALD TiN/ HfO2/IL/Si sub. & $7 \mathrm{~s}$ & - \\
\hline
\end{tabular}

Table 1: Split conditions of HRTEM samples.

(a)

(b)

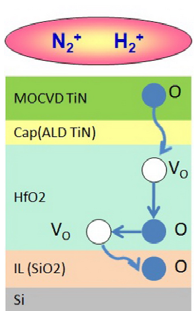

(c)

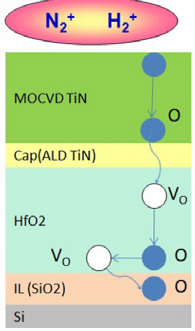

(d)

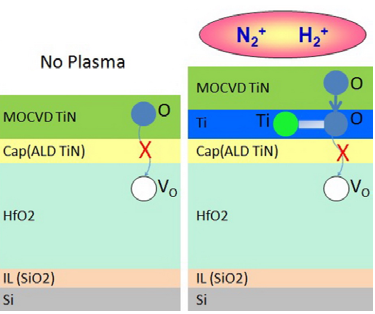

Figure 9: Schematic of the effect of plasma treatment on EOT.

accelerate interfacial oxide formation and increase equivalent oxide thickness (EOT) due to oxygen diffusion of MOCVD TiN film into IL layer by the action of plasma treatment.

\section{Acknowledgments}

This work was supported by the Ministry of Science and Technology of China under Contract $2009 Z X 02035$ through the $22 \mathrm{~nm}$ Technology Program.

\section{References}

1. BaohongC, Min C, Ramgopal R (1999) The impact of high-gate dielectrics and metal gate electrodes on sub-100 nm MOSFET's. IEEE Trans Electron Devices 7: 1537 .

2. Yeo YC, King TJ, Hu CM (2002) Metal-dielectric band alignment and its implications for metal gate complementary metal-oxide-semiconductor technology. J Appl Phys 12: 7266

3. Chudzik M, Doris B, Mo R, Sleight J (2007) High-performance high-kappa/ metal gates for $45 \mathrm{~nm}$ CMOS and beyond with gate-first processing. IEEE Symposium on VLSI Technology 194.

4. Auth C, Buehler M, Cappellani A, Chi-hing C, Ding G, et al. (2008) "45 nm high-k+metal gate strain-enhanced transistors". Intel Technology Journal 2: 77.

5. Kadoshima M, Matsuki T, Miyazaki S, Shiraishi K, Chikyo T, et al. (2009) Effective-Work-Function Control by Varying the TiN Thickness in Poly-Si/TiN Gate Electrodes for Scaled High- CMOSFETs. Electron Device Letters IEEE 5: 466.

6. Westlinder J, Sjoblom G, Olsson J (2004) Variable work function in MOS capacitors utilizing nitrogen-controlled $\mathrm{TiN}$ x gate electrodes. Microelectronic Engineering 75: 389

7. Liang Q, Xu QX (2011) A new EOT shrinking mechanism in TiN/HfLaON HKMG MOSFET: Experimental and ab-initio study. ISDRS: 2.

8. Wu L, Yu HY, Li X, Pey KL, Hsu KY, et al. (2010) Investigation of ALD or PVD (Ti-rich vs. N-rich) TiN metal gate thermal stability on $\mathrm{HfO}_{2}$ high-K. VLS Technology Systems and Applications (VLSI-TSA) 90.

9. Wu L, Yu HY, Li X, Pey KL, Pan JS, et al. (2010) Thermal stability of TiN meta gate prepared by atomic layer deposition or physical vapor deposition on $\mathrm{HfO}_{2}$ high-K dielectric 11: 113510

10. Lee SR, West AM, Allerman AA (2005) Effect of threading dislocations on the Bragg peak widths of GaN, AIGaN, and AIN heterolayers. Appl Phys Lett 24: 241904.

11. Ranade P, Choi YK, Ha D, Agarwal A, Ameen M, et al. (2002) "Tunable work function molybdenum gate technology for FDSOI-CMOS". IEDM Tech Dig: 363-366.

12. Smoluchowski R (1941) "Anisotropy of the electronic work function of metals." Phys Rev 60: 661-674.

13. Bai WP, Bae SH, Wen HC, Mathew S, Bera LK, et al. (2005) Three-Laye Laminated Metal Gate Electrodes With Tunable Work Functions for CMOS Applications. IEEE Electron Device Letters 4: 231-233.

14. Goncharova LV, Dalponte M, Starodub DG, Gustafsson T (2006) Oxygen diffusion and reactions in Hf-based dielectrics. Applied Physics Letters 4 044108.

15. Kai H, Xueli M, Hong Y, Wenwu W (2013) Modulation of the Effective Work Function of TiN Metal Gate for pMOS Application. Journal of semiconductors 8: 086002 